\title{
VISCOUS FLOW STRUCTURE INTERACTION
}

by

Anton10 Huerta, Graduate Student

and

Wing Kam Liu, Member, ASME

Department of Mechantca1 Engineering Northwestern Dniversity

Evanston, 1111no1s 60201

To be presented at the "Symposium on Analysis and Testing of Vibrational and Sefsml c Response of Fluid-Structure Systems," 1987 ASME PVP Conference to be held in San Diego, Callforn1a, June 28 to July 2, 1987. 


\begin{abstract}
Considerable research activities in pibration and seismic analysis for varlous fluid-structure systems have been carried out in the past two decades. Most of the approaches are formulated with1n the framework of fintte elements, and the majority of work deals with inviscid flulds. However, there has been little work done in the area of fluid-structure interaction problems accounting for flow separation and nonlinear phenomenon of steady streaming. In this paper, the Atbitrary Lagrang1an Eulerian (ALE) fin1te element method is extended to address the flow separation and nonifnear phenomenon of steady streaming for arbitrarily shaped bodies undergoing large periodic motion in a v1scous flu1d. The results are designed to evaluate the fluid force acting on the body; thus, the coupled rigid body-viscous flow problem can be simplified to a standard structural problem using the concept of added mass and added damping. Formulas for these two constants are given for the particular case of a cylinder immersed in an infintte viscous fluid. The finite element modeling is based on a pressure-velocity mixed formulation and a streamline upwind Petrov/Galerkin technique. All computations are performed using a personal computer.
\end{abstract}




\section{Introduct1on}

Th1s paper describes the application of the Arb1trary Lagrang1an Eulerian (ALE) viscous fluid formulation [1] to evaluate the fluid force acting on a vibrating rigid body. The motion of an osc1llating body is governed by 1ts mass, the stiffness of the spring, the damping coefficient, the surrounding viscous fluid and the external force acting on the solid (see Figure 1). In current engineering practice, the influence of an inviscid fluid is taken into account by means of a hydrodynamic mass or an added mass $[2,3]$. When dealing w1th viscous flulds, the added aass cannot describe properly the force acting on the body, thus, an added damping term is needed. To avold coupling between the spring-mass system and the nonlinesr Navier-Stokes equations, the equation of motion for the rigid body is simplified to:

$$
M \ddot{d}+c d+K d=F_{\text {ext }}-F_{f}
$$

where $\mathrm{M}, \mathrm{C}, \mathrm{K}, \mathrm{F}_{\text {ext }}$ and $\mathrm{d}$ are the mass, structural damping coeffieient, structural spring constant, external force, and displacement, respectively, of the rigid body. A superposed dot denotes material tiwe differentation. $F_{f}$ is the resultant fluld force acting on the body (1.e., without actualiy modeling the v1scous fluid) and it ill asauned of the form:

$$
F_{f}=M_{a d} \ddot{d}+C_{a d} d
$$

where $M_{a d}$ and $C_{a d}$ are the added mass and added damping coefficients, respectively. Substituting Eq. (2) Into Eq. (1) y1elds

$$
\left(M+M_{a d}\right) \ddot{d}+\left(C+C_{a d}\right) d+K d=F_{\text {ext }}
$$

which can be solved readily since it is a standard one degree of freedoa atructural dynamic equation, provided $M_{a d}$ and $C_{a d}$ are evaluated properiy. Mad and $\mathrm{C}_{\mathrm{ad}}$ can be estimated by prescribing to the rigid body a sinusotdal motion

$$
d=\sin w t
$$

where $\mathrm{o}$, w and $t$ are the amplitude of the motion, c1rcular frequency and 
t1me, respectively.

In the next section, the governing equations for a viscous flutd subject to a large boundary motion, such as given by Eq. (4), are formulated. In Section 3, the computational procedures for $M_{\text {ad }}$ and $C_{\text {ad }}$ using the ALE method are described. An application to a clrcular cylinder osciliating in a viscous fluid is given in Section 4. The concluding remarks ate presented in Section 5. 


\section{Governing Equations for V1scous Fluids in ALE Formulation}

Cons1der a flu1d doma1n $R$ described by mater1al, spatial, or referential coordinates which are denoted by $\underset{\sim}{x}, \underset{\sim}{x}$ and $\underset{\sim}{\not}$, respectively. Throughout this paper, repeated indices denote a summation over the number of space dimensions (NSD). The spatial derivative (1.e., derivative with respect to $\underset{\sim}{x}$ ) 1 s designated by a comma followed by a subscript while the material, spatial and referential time derivativen of an arbitraty function $f$ are represented by ${ }^{f}, t[\underset{\sim}{x}], f_{,}^{f} \underset{\sim}{x]}$ and $f, t[\underset{\gamma}{ }]$, respectively. The continuity and Navier-stokes equations for a Newtonian, 1sothermal fluid are $[4,5]$

$$
\frac{1}{B} P_{, t}\left[x_{N}\right]+v_{1,1}=0
$$

and,

$$
v_{1, t[X]}+c_{1} v_{1,1}=-\frac{1}{p} p_{, 1}+v\left(v_{1,1}+v_{1,1}\right)_{, j}+b_{1}
$$

Where B is the bulk modulus of the fluid, P is the thetmodynamic pressure: $\underset{\sim}{v}$ is the material velocity vector; $p$ is the fluid density; $v$ is the kineatic viscosity; $\underset{\sim}{b}$ is the body force vector; and $\underset{\sim}{\mathrm{c}}$ is the convective velocity vector. The convective velocity is defined as

$$
c_{1}=v_{1}-\hat{v}_{1}
$$

where $\hat{z}$ is the mesh velocity vector which is arbitrarily ehosen depending on the part1cular problem $[6,7]$.

The variational equations associated with Eqs. (5) are $[7,8]$

and,

$$
\int_{R_{x}} \delta P \frac{1}{B} P, t[\underset{\sim}{X}] d R_{x}+\int_{R_{x}} \delta P v_{1,1} d R_{x}=0
$$


$\left.\left.\int_{R_{x}} \delta \tilde{v}_{1} v_{1, t}\right]_{X}\right] d R_{x}+\int_{R_{x}} \delta \tilde{v}_{1} c_{1} v_{1, j} d R_{x}-\int_{R_{x}} \frac{1}{\rho}\left(\delta v_{1}\right), 1 P d R_{x}+$

$\int_{R_{x}} \frac{v}{2}\left[\left(\delta v_{1}\right)_{, j}+\left(\delta v_{j}\right)_{, 1}\right]\left[v_{1, j}+v_{j, 1}\right] d R_{x}-\int_{R_{x}} \delta \tilde{v}_{1} b_{1} d R_{x}-\int_{\partial R_{x}^{h}} \delta v_{1} h_{1} d S=0$

The weighting function $6 \mathrm{P}$ in $(7 \mathrm{a})$ is chosen to be discontinuous across the interelement boundaries and constant inside the element. The same function 6P is used for the presaure interpolation completing a Galerikin formulation. On the other hand, the weighting function $\delta \tilde{v}_{1}$ in (7b) is composed of two terms: the first one, $6 \mathrm{v}_{1}$, 15 continuoun within the elements and across their boundaries; and the second term is the discontinuous streamilne contribution. In Eq. (7b) a Petrov/Galerkin formulation is used becauge the interpolation functions for the velocities are 11near combinations of the continuous term, $\delta \mathrm{v}_{1}$. Hughes and Brooks $[9]$ present a detalled discussion on the assumptions required for obtaining Eq. (7b), it should be noted that the cont1nuous part of the velocity velghting function, $\delta v_{1}$, sust satisfy $\delta v_{1}=0$ on the boundary where the velocity field is prescribed. The

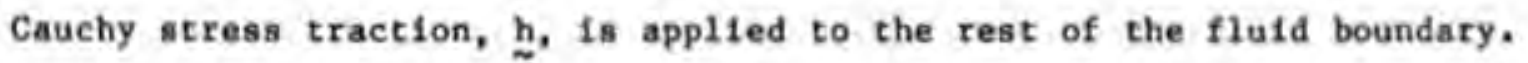

The spatial discretization of Eqs. (7) via f1nite elements leads to the following two systems of matrix equations:

$$
\begin{aligned}
& \underset{\sim}{\mathrm{M}} \underset{\sim}{\mathrm{P}}+\underset{\sim}{\mathrm{g}^{\mathrm{T}}} \underset{\sim}{\mathrm{v}}=\underset{\sim}{0}
\end{aligned}
$$

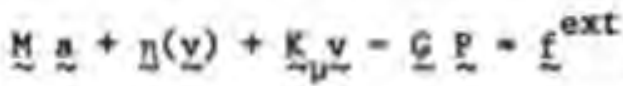

where $\underset{\sim}{P}$ is the pressure-masa matrix; $\underset{\sim}{M}$ is the fluid mass matrix; and $\underset{\sim}{v}$ and $\underset{\sim}{\mathrm{P}}$ are the vectors of unknown nodal values for velocity and pressure, respectively. $\underset{\sim}{\mathrm{p}}$ is the material time derivative of $\mathrm{P} ; \underset{f}{\mathrm{a}}$ is the time derfvative of the material velocity vector, $\underset{\sim}{\mathbf{v}}$, holding the reference fixed; (1.e., $\&$ fixed), $\cap$ is the generalized convective velocity vector: $f^{\text {ext }}$ is the 
total external load vector applied to the fluid, $\underset{\sim}{\underset{\sim}{K}}$ is the fluid viscosity matrix and $\underset{\sim}{G}$ 1s the divergence operator matrix.

Remark 1: If the fluid is assumed Incompressible, Eqs. (8) are further simplified to

$$
\begin{aligned}
& \underset{\sim}{\mathrm{G}^{\mathrm{T}}} \underset{\sim}{\mathrm{v}}=\underset{\sim}{0}
\end{aligned}
$$

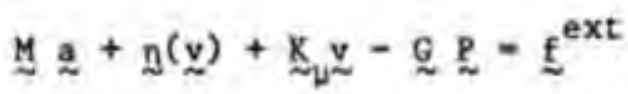

Remark 2: A predictor-multicorrector algorithm described in Refs. $[1,10]$ is used to solve efther Eqs. (8) or (9). Tro passes (1.e., one 1teration) are used to insure stability and account for the upwinding effects on the mass term.

In order to determine the dimenalonless parameters governing the boundary value problem, the incompressible fleld equations (1.e., Eqs. (5)) are written 1n a nondimensional fora [11]. The length scale used is the dimension b of the rigid body in the motion direction (see Fig. 1). The time scale $1 \mathrm{~s} \mathrm{w}^{-1}$ and the characteristic velocity $\mathrm{y}_{0}=\mathrm{s}_{0} \mathrm{w}$ is chosen to scale the velocities. Recal1, from Eq. (4), that $v_{0}$ is the amplitude of the prescribed velocity. Finally, $\mu v_{0} / \mathrm{b}$ is the pressure scale where $\mu$ is the absolute viscosity of the fluid. In this analysis, the gravity is perpendicular to the plane under constderation (1.e., the body forces are zero) and Incompressibility of the fluid is assumed. Consequently, scaling with $b, w^{-1}$ and $v_{0}$, Eqs. (5) become:

$$
\begin{aligned}
& \bar{v}_{1,1}=0 \\
& \left.\bar{v}_{1, \bar{t}|\gamma|}+\beta \bar{c}_{j} \bar{v}_{1,1}-\frac{1}{R_{w}} \mid\left[\bar{v}_{1,1}+\bar{v}_{1,1}\right]_{, j}-\bar{p}_{, 1}\right]
\end{aligned}
$$

where the superscripted bars denote dimensionless functions, all the spatial 
derivatives are with respect to dimensionless coordinates $\bar{x}_{1}=x_{1} / b$, s 1 s an amplitude ratio parameter and $R_{w}$ is the frequency Reynolds number. However, for Inviscid fluids (1.e,, $R_{\text {w }}$ approaches to infinity) the right hand side of Eq. (10b) w11 become zero and the result1ng equilibrium equations are not the classical Euler equations. Hence, a different pressure scaling is introduced. The characteristic pressure is defined as pbow ${ }_{0}$, and Eq. (10b) becoses

$$
\bar{v}_{1, \bar{c}[\chi]}+B \bar{c}_{j} \bar{v}_{1, j}-\frac{1}{R_{w}}\left[\bar{v}_{1, j}+\bar{v}_{j, 1}\right]_{, j}-\bar{P}_{, 1}
$$

The solution characteristics of this problem are governed by the two parameters in Eqs, (10) and $a$, which is associated with the geometry. They are defined as:

$$
a=\frac{D}{b}, B=\frac{s}{b}, \text { and } R_{w}=\frac{b^{2} w}{v}=\frac{R e}{B}
$$

where D is the diameter of the outer boundary of the fluid doantin and Re is the Reynolds number $\left(1 . e .\right.$, Re $\left.-v_{0} b / v\right)$. Frow Eq. $(10 b)$ one can notice that $\beta$ and $R_{w}$ are associated with convection and diffusion, respectively. Therefore, for "sma $11^{*} B$ and $R_{w}$ the diffusion effects govern the velocicy f1eld; whereas, with "1arge" $B$ and $R_{w}$ the convection dominates. In order to define the boundary value problem completely, the dimensionless boundary conditions and mesh velocities are given as follows. Velocity conditions need to be specified on the boundaries, whereas, the pressure needs to be fixed at one point in the fluid domaln to obtain a pressure datum. On the outer cylinder and in the vertical direction along the symetry ilne the fluid velocity is zeto while on the rigid body the dimensionless form of the boundary condition, defined by Bq. (4), is given by: 


$$
\begin{aligned}
& \bar{v}_{1}\left(\bar{x}_{11}+\bar{d}(\bar{t}), \bar{x}_{21}\right)=\cos \bar{t} \\
& \bar{v}_{2}\left(\bar{x}_{11}+\bar{d}(\bar{t}), \bar{x}_{21}\right)=0
\end{aligned}
$$

where

$$
\bar{d}(\bar{t})=B \sin \bar{t}
$$

and $\bar{x}_{1}$ are the dimensionless coordinates for the contour of the inner body. The Influence of $B$ on the equilibrium equation and on the motion of the Inner boundary suggests that a perturbation technique can be used to solve this problem, see Ref. [11]. However, such a procedure is only valid for small $B$. Converse1y, the Arb1trary Lagranglan Eulertan formulat1on used here does not restrain the magnitude of the amplitude rat1o, $B$.

Unless a perturbation technique is used, the velocity conditions at the sol1d body boundary require a Lagrang1an description. That 18 , the mesh must follow the particle movement at this boundary. However, elsewhere and princ1pally away from the osc1llating body, a Eulerian description is better sulted because a fixed reference through which the fluld moves allows atrong distortions due to the flow. Finally, in order to evaluate the boundary effect, the density of elements must increase in the vicinity of the moving sol1d. These requirements can be fulfilled by using an ALE formulation: at the surface of the osc1llating body the mesh motion ta prescribed equal to the particle motion, (2) away tron the nolid the sesh is fixed and (3) in between, a transition zone is defined wth an arbitrarily prescribed besh motion. The mesh velocities can be defined as:

$$
\hat{v}_{1}= \begin{cases}\frac{\mathrm{s}}{2}\left[1+\cos \left(\frac{l \pi}{\mathrm{L}}\right)\right] \omega \cos \omega t & \text { if } \ell<\mathrm{L} \\ 0 & \text { if } \ell>\mathrm{L}\end{cases}
$$


and

$$
\hat{v}_{2}=0
$$

where $\ell$ is the horizontal distance between the referential point (mesh node) and the rigid body surface, and $L$ is an arbitrar1ly chosen transition length. Finally it is noted that a stream11ne upwind Petrov/Galerkin formulation 1s required because of the relative inportance of the convective effects. In general, the convective effect is increased in the vicinity of the noving sol1d. Therefore, for Reynolds numbers greater than 50, upwinding is necessary. A temporal, rather than apatial criterion [12], 1s selected for the perturbation of the weighting function because of the time dependency of $\underset{\sim}{\mathrm{c}}$ and the Increased convection which can occur in the sallest elements. 


\section{Deternination of the Added Mass and Damping Coeffictents}

The evaluation of $\mathrm{M}_{\mathrm{ad}}$ and $\mathrm{C}_{\mathrm{ad}}$ is similar to the procedure described in Ref. [3]. That 1s, the body motion $1 \mathrm{~s}$ prescribed and the fluid force, $F_{f}$, acting on the solid is evaluated. S1nce acceleration and velocity cannot be applfed Independently of each other (I.e., set the acceleration equal to one and the velocity to zero, or vice-versa) the prescribed sinusotdal motion defined by Eq. (4) 1 s chosen. In this manner, $F_{f}$ can be expanded uaing a Pourier serfes; and consequentiy, the term in phase with the acceleration is separated from the one in phase with the velocity. From these two teras, the added mass and damping can be computed. The Fourler decomposition of $F_{f}$ is as follows :

$$
F_{f}=A_{0}+\sum_{n=1} A_{n} \cos (n \omega t)+\sum_{n=1} B_{n} \sin \left(n_{\omega} t\right)
$$

where,

$$
\begin{aligned}
& A_{0}=\frac{1}{2 \pi} \int_{0}^{2 \pi} F_{f} d t, \\
& A_{n}=\frac{1}{\pi} \int_{0}^{2 \pi} F_{f} \cos (n \omega t) d t, n=1, \ldots= \\
& B_{n}=\frac{1}{\pi} \int_{0}^{2 \pi} F_{f} \sin (n \omega t) d t, n=1, \ldots=
\end{aligned}
$$

If the 1nitial assumption that $F_{f}$ is a 11near coabination of acceleration and velocity is true, only two coefficients $\left(A_{1}\right.$ and $\left.B_{1}\right)$ are nonzero. Obviously, these coefficients are the ones associated wth the same frequencies as the prescribed acceleration and velocity. Once $A_{1}$ and $B_{1}$ are computed from EqB. (16b) and (16c), the added anss and damping are determined by 


$$
M_{\text {ad }}=-\frac{B_{1}}{s_{0} \omega^{2}}
$$

and

$$
c_{a d}=\frac{A_{1}}{s_{0} \omega}
$$

respectively. Or, in dimensionless form, using the scaling factors defined in the previous section:

$$
\bar{M}_{\text {ad }}=-\frac{B_{1}}{s_{0} \omega^{2}} \frac{1}{\rho b^{2}}
$$

and

$$
\vec{c}_{a d}=\frac{A_{1}}{s_{0} \omega^{2}} \frac{1}{\rho b^{2} \omega}
$$

W1th reference to $\mathrm{Fig} .1$, the computation of $\mathrm{P}_{\mathrm{f}}$ at every Instant can be obtalned by integrating both the thermodynamic pressure $P$ and the viscous ahear forces around the body. The added mass and damping paraneters can then be computed; and the rigid body-vincous tlow interaction problea is siaplifled to Eq. (3). 


\section{Numer1cal Examples}

Numerical results are obtalned for a circular body osc11lating with a sinusoidal motion. The c1rcular body shape is chosen in order to compare the hydrodynamic force obtalned using th1s method with the Fritz formulas [2]. The latter is valid only for a cylinder imersed in inviscid fluids. The method described in this paper can account for an arbitrary geometry in a viscous fluid.

A11 calculations are performed in single precision (32 bits per word) on a $640 \mathrm{k} 1$ lobyte IBM-XT personal computer with an 8087 mathenatical processor.

The dimensionless parameter $\alpha$ is set to 30 throughout this analya1s. The Influence of the other two parameters governing the problem, namely 8 and $R_{w}$, is assessed by varying them in the following fashion: the anplitude ratio parameter, B, is taken equal to $0.1,0.4,0.75$ and 1.0 ; the frequency Reynolds number, $R_{W}$, varies from 20 to infinity. In fact, these different cases represent a Reynolds number $\left(R e=R_{w} B\right)$ which ranges between 2 and infinity.

The dimensionlese time-step used in the computation is usually given by $w \Delta t=\frac{\pi}{20}$; however, in the cases highly domlnated by diffusion or convection 1t is reduced to $\frac{\pi}{28}$. As shown in F1g. 2 ; the 224 -node mesh consists of $1954-$ node elements (constant pressure elements). Finally, the transition length for the mesh movement does not influence the results if $\mathrm{L}>9 \mathrm{~s} 0$.

F1gures 3 and 4 show the 1nstantaneous streamlines tor $8=0.1$ and two frequency Reynolds numbers: 20 and 500. As expected, for high $R_{w}$ most of shearing occurs in a small layer surrounding the rigid body. At $t-\frac{\pi}{2} a$ vortex elearly appears, but it disappears alnost Instantaneously. However, for large $\beta$ and $R_{w}$ the vortex does not disappear and vortex shedding occurs, see Fig. 5 where $B=1.0$ and $R_{W}=1000$. Although the purpose of this study is to compute the flu1d force on the sol1d, $81 \mathrm{~g} .5$ clearly suggests that a vortex 
shedding analysis can be conducted with the present method if a finer mesh is used away from the solld boundary $1 \mathrm{n}$ order to capture the vortex movement.

The pressure distribution around the rigid body for $B=0.1$ and $R_{w}=20$ and 500 (1.e., $\operatorname{Re}=2$ and 50 , respectively) is presented in Fig. 6. One can observe that the angle $\theta$ for the stagnation point decreases (1.e., teading to $\frac{\pi}{2}$ ) as $R_{w}$ is increased at $t=0$, and that the pressure amplitude decreases as $R_{w}$ 1ncreases. The last phenomenon is also observed in Fig. 7, where the variation of the fluld reaction $\left(F_{f}\right), 18$ plotted versus $t 1 m e$. The relative Importance of the added mass and damping via the decomposition of $F_{f}$ is also depicted In F1g. 7. For 1nstance, the ratio of damping to mass force decreasen from $75 \%$ at $R_{w}=20$ to $15 \%$ at $R_{w}=1000$.

It is 1mportant to not1ce that both added mass and damping are frequency dependent for v1scous flulds. F1gures 8 and 9 show the varlations of the two parameters with $R_{W}$. From these figures, it can be concluded that added mass 18 Independent of the amplitude ratio $B$, while the added damping varies with B. As expected, for all B, the added damping goes to zero and the added mass remains constant as $R_{w}$ goes to infinfty. The computed added mass for $R_{v}$ - Is 0.8169 which compares well (3.8z relative error) with Fritz's approximation $(0.7871)$, given the coarseness of the mesh. If the added mass results are interpolated using Pritz formulas for the 11-itt, the following equation is obtafned:

$$
\bar{M}_{\text {ad }}=5.992 \mathrm{R}_{W}^{-0.643}+0.787
$$

with a correlation of 98.3z. Obviously, this correlation would be improved if the sctual computed wass for $\mathrm{R}_{\mathbf{\alpha}}=$ - was taken; however, Fritz formulas are the simplest and most extended way to approximate the value of the added mass for an Inviacid fluid. The added damping interpolation ia a little more complicated because the added viscosity is a function of B. However, Pig. 9 
suggests that $\beta$ only Influences the slope of the Log-Log curve. Hence, the following 1nterpolation is obtalned:

$$
\bar{C}_{\text {ad }}=1.88\left(R_{\alpha} / 9.42\right)^{0.3618-0.618}
$$

and the curves plotted in FIg. 9 are derived from the direct application of Eq. (20) for different parameters $B$ and $R_{w}$. 


\section{Conclusions}

Th1s paper shows that Arb1trary Lagrangian Eulerian techniques are applicable to vibration and se1smic analysis for flu1d-structure systems. An ALE formulation is presented for a viscous fluid subject to a large boundary motion. Because of the use of a pressure-velocity mixed formulation, and the improvements in accuracy of the streamline upwind Petrov/Galerkin techniques, the computer program is implemented on a personal computer.

The concept of added mass and damping is used to simplify the rigid bodyv1acous flow 1nteraction problem 1nto a standard structural dynamic analysis. When the fluid force on the solld is expanded via Fourler analysis, only the added mass and damping terms are nonzero. The hydrodynamic mass computed for the 1nviscid case agrees well with the Fritz formula.

A parametric analysis shows that $M_{a d}$ is only a function of the frequency Reynolda number, and a simple formula is presented for the particular geocetry studied here. On the other hand, the added damping depends on the frequency Reynolds number and the amplitude ratio of the notion. A simple formula is also obtalned for the predietion of $\mathrm{C}_{\mathrm{ad}}$ in this particular problem.

\section{Acknowledgements}

The financial assistance provided to Antonio Huerta by a Catalan Fellowship (CIRIT BE84-26) is gratefully acknowledged. The support of Wing K. L1u by the National Science Foundation is also gratefu11y scknowledged. 


\section{References}

1. LIu, W. K. and Gvildys, J., "Fluid-Structure Interaction of Tanks with an Eccentr1c Core Barre1, “ Computer Methods 1n Applied Mechanics and Eng1neer1ng, Vol. 58, pp. 51-57, 1986.

2. Fritz, R. J., "The Effect of Liquids on Dynamic Motione of Immersed Sol1ds," Journal of Englneering for Industry, ASME, Vol. 94, pp. 167-173, 1977.

3. L1U, W. K., Lam, D. and Belytschko, T., "F1nite Element Method for Hydrodynamic Mass with Nonstationary Fluid," Computer Methods in Applied Mechantes and Engineering, Vol. 44, pp. 177-211, 1984.

4. Lfu, W. K. and Ma, D., "Computer Implementation Aspects for Fluid Structure Interact Ion Problems," Computer Methods in App11ed Mechan1cs and Engineering, Vol. 31, pp. 129-148, 1982 .

5. LIU, W. K. and Chang, H. G., "Effleient Computational Procedures for Long-T1me Duration Flu1d-Structure Interaction Problems," Journal of Pressure Vesse1 Technology, A3MB, Vo1. 106, pp. 317-322, 1984.

6. Hughes, T. J. R., L.1u, W. K. and Z1mmerman, T. K., "Lagrangian-Eulerian Finite Element Formulation for Incompressible Viscous Flous," Computer Methods in Applied Mechanics and Engineering, Vol, 29, pp. $329-349,1981$.

7. Liu, W. K., Belytschko, T. and Chang, H., "An Arbitrary LagrangianEulerian Finite Element Method for Path-Dependent Materials, - Conputer Methods in Applled Mechanics and Engineering, Vol. 58, pp. 227-246, 1986.

8. Brooks, A. N. and Hughes, T. J. R., "Streamline Upwlnd/Petrov-Galerk1n Pormulations for Convection Dominated Flows with Particular Emphasis on the Incompressible Navler-Stokes Equations," Computer Methods in Applied Mechantes and Englneering, Vol. 32, pp. 199-259, 1982 .

9. Hughes, T. J. R. and Brooks, A. N., "A Theoret1cal Framework for PetrovGalerkin Methods with Discontinuous Weighting Functions: App11cation to the Streamline-Upwind Procedure," In F1nite Elements in Fluide, Vol. 4, Gallagher, R. H., Norrie, D. H., Oden, J. T. and Zlenkiewlez, O. C., eds., John Wiley and Sons, L.td., pp. 47-65, 1982.

10. L1u, W. K. and Chang, B. C., "A Method of Computation for Flu1d Structure Interaction," Journal of Computers and Structures, Vo1. 20, No. 1-3, pp. $311-320,1985$.

11. Pattant, P. G. and O1son, M. D., "Periodic Solutions of Rigid BodyViscous Flow Interaction," preprint.

12. Hughes, T. J. R. and Tezduyst, T. E., "Finite Element Methods for FirstOrder Hyperbolic Systems with Particular Bmphasis on the Compressible Euler Equations," Computer Methods in Applied Mechanics and Engineering, Vol. 45, pp. 217-284, 1984. 


\section{F1gure Captions}

Figure 1. Problem Statement.

Figure 2. Mesh d1scretization a) Complete Doma1n, b) Imposed Mesh Distortion.

Figure 3. Instantaneous Stream 11 nes $f_{g_{\pi}} B=0.1$ and $R_{w}=20$ at $\left.a\right) t=0$, b) $\left.t=\frac{\pi}{4}, c\right) t=\frac{\pi}{2}$, d) $t=\frac{3 \pi}{4}$.

F1 gure 4. Instantaneous Streamlines $f g r s=0.1$ and $R_{w}=500$ at a) $t=0$, b) $t=\frac{\pi}{4}$, c) $t=\frac{\pi}{2}$, d) $t=\frac{3 \pi}{4}$.

F1gure 5. Vortex Fgrmation for $B=1.0$ and $R_{w}=1000$ at $a$ ) $t=\frac{\pi}{2}$,
b) $t=\frac{3 \pi}{4}$.

F1gure 6. Pressure Distribution around the sol1d for $\beta=0.1$ and a) $R_{w}=20$, b) $\mathrm{R}_{\mathrm{w}}=500$.

Figure 7. Fluid Force versus Time for the Decomposition of $F_{f}$ in Mass and Damping terms with $B=0.1$.

F1gure 8. Dimensionless Mass $\left(\vec{M}_{\text {ad }}\right)$ versus Frequency Reynolds Number $\left(R_{w}\right)$.

Figure 9. Dimensionless Damping $\left(\bar{C}_{\text {ad }}\right)$ versus Frequency Reynolds Number $\left(\mathbb{R}_{w}\right)$. 


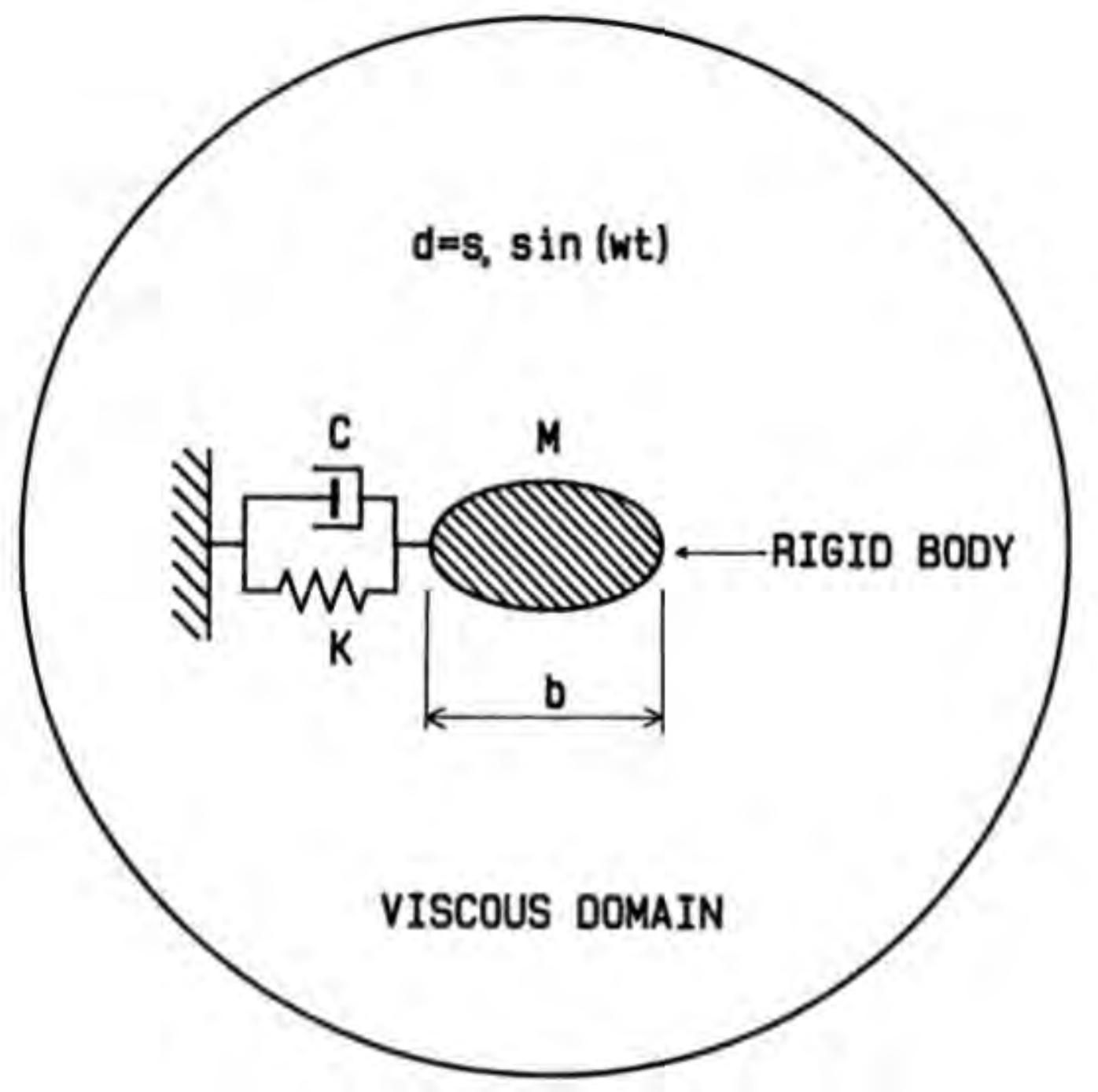

Fig I 

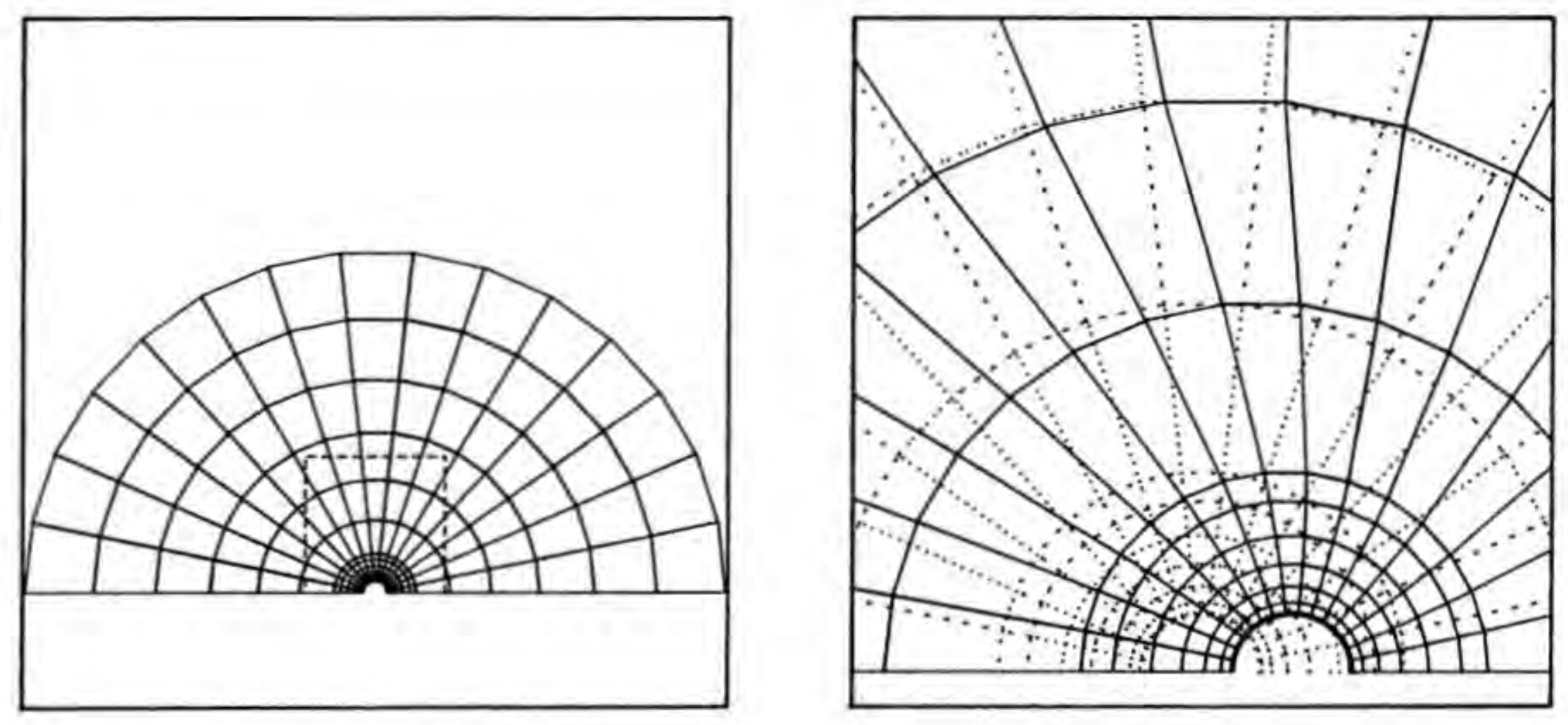

in 2 

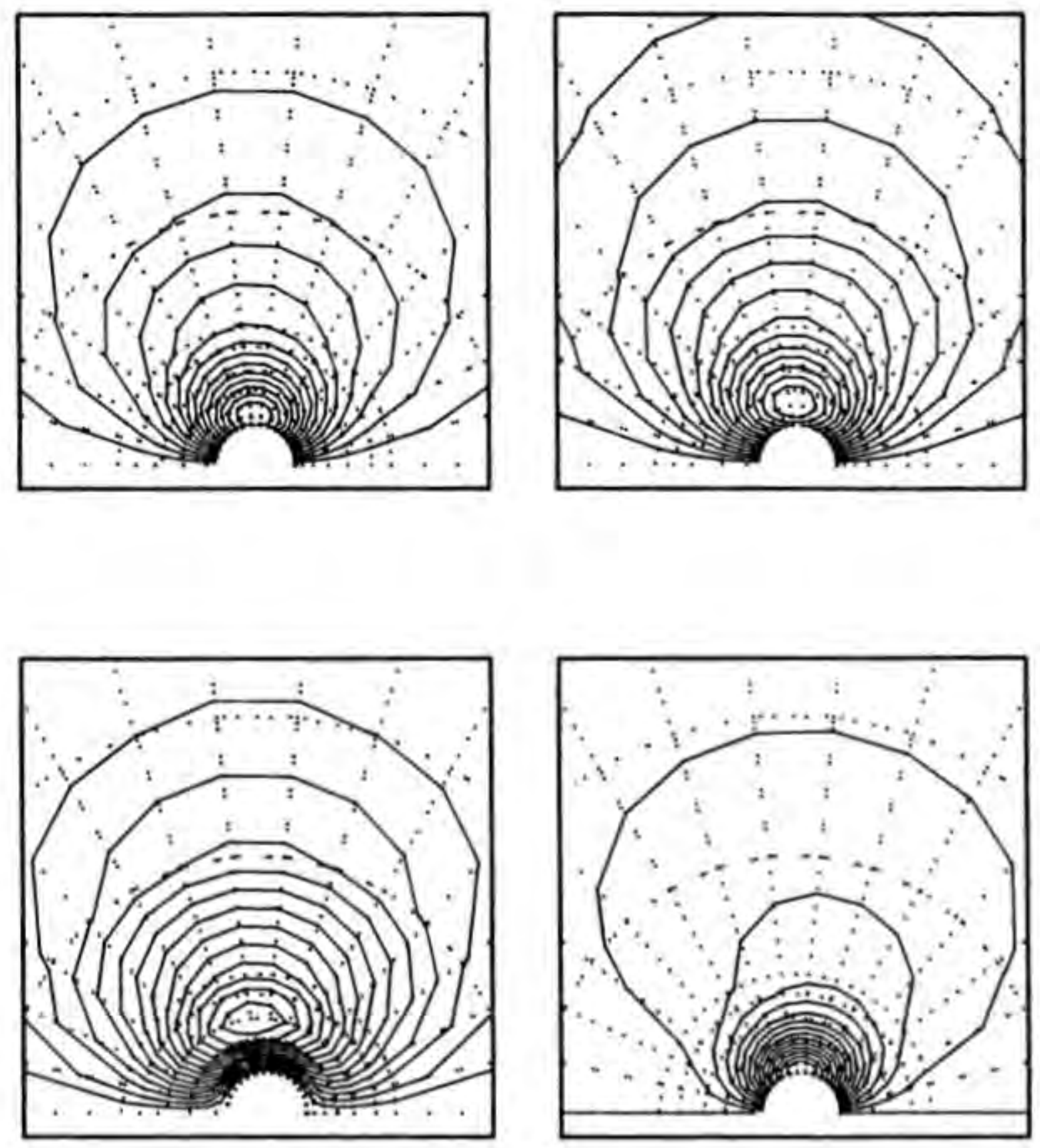

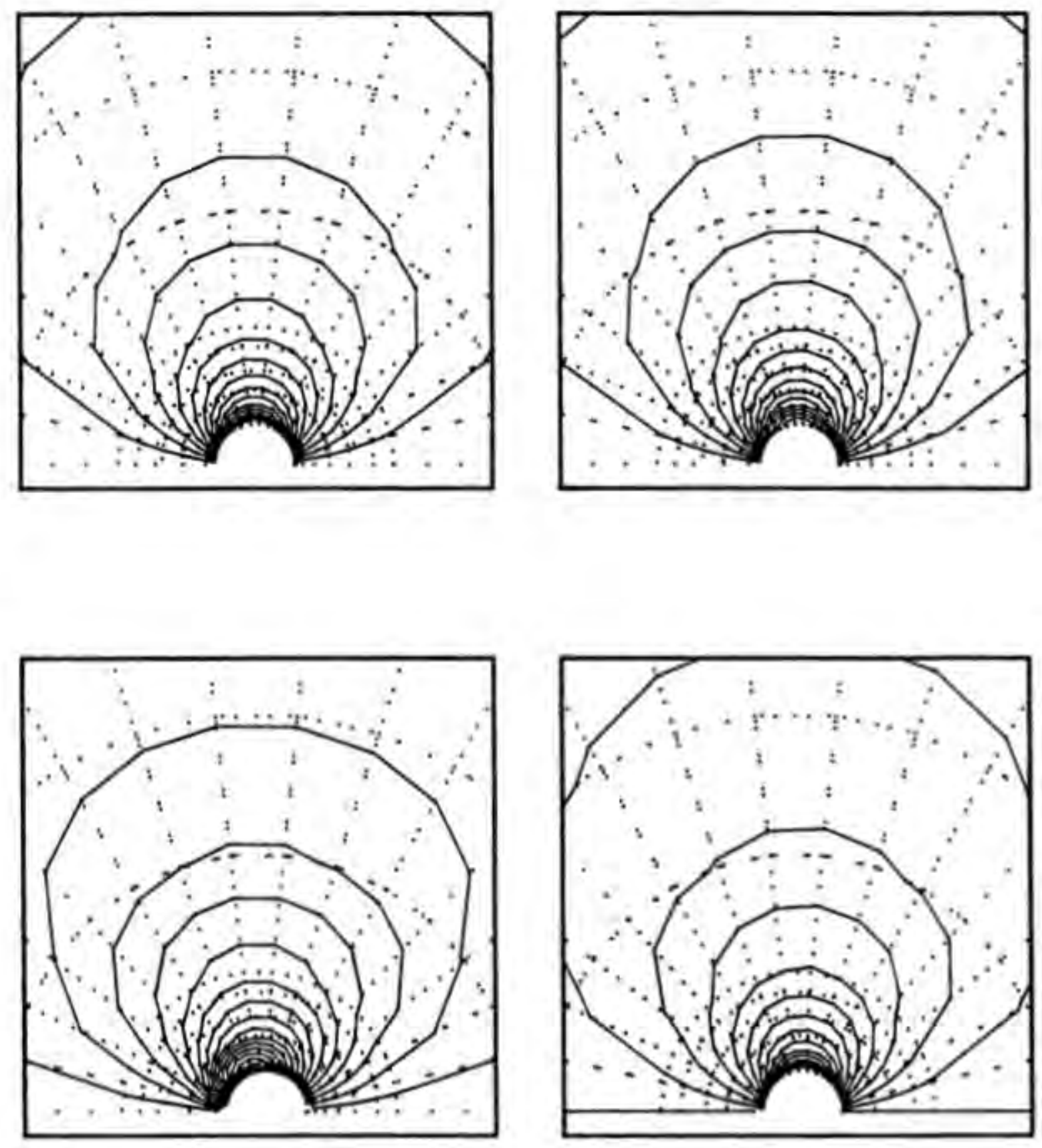

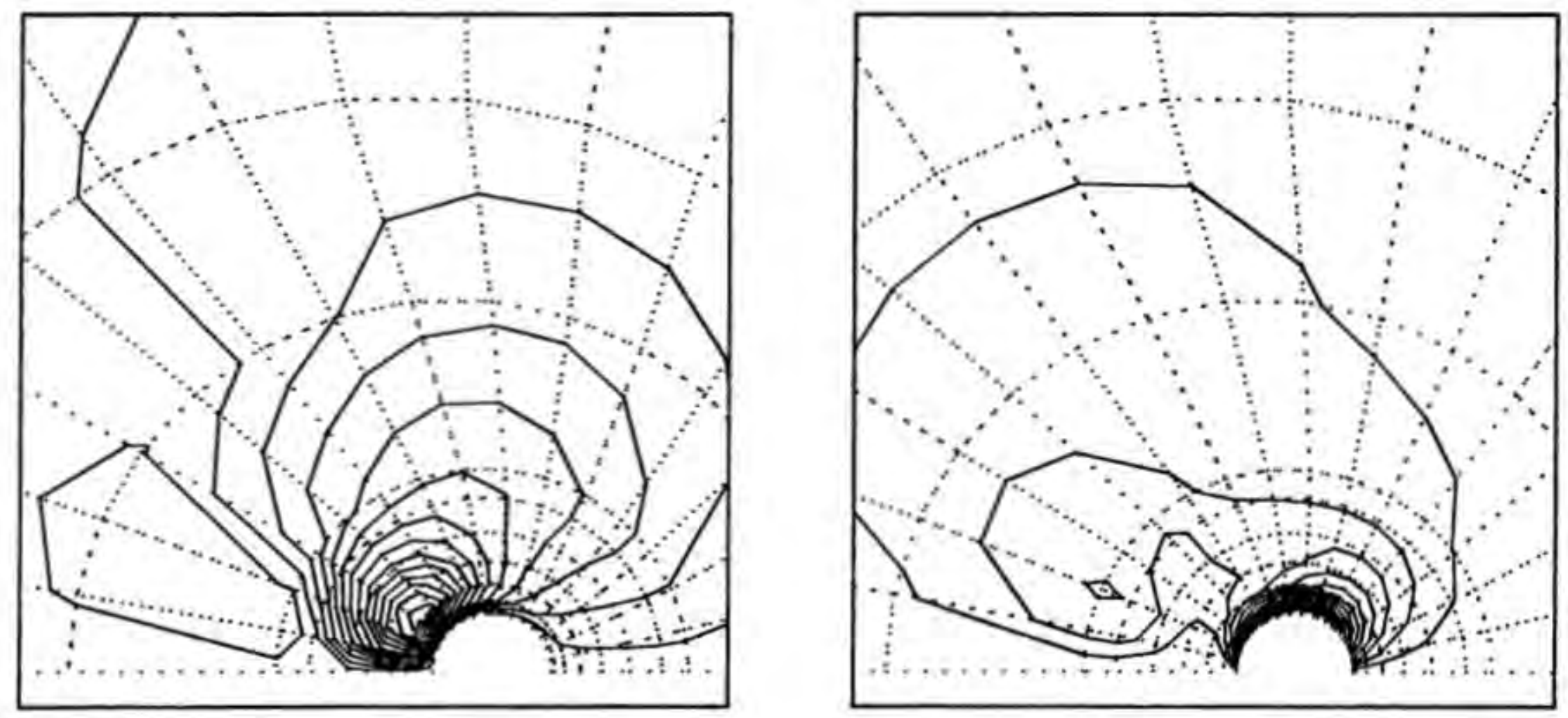

75 

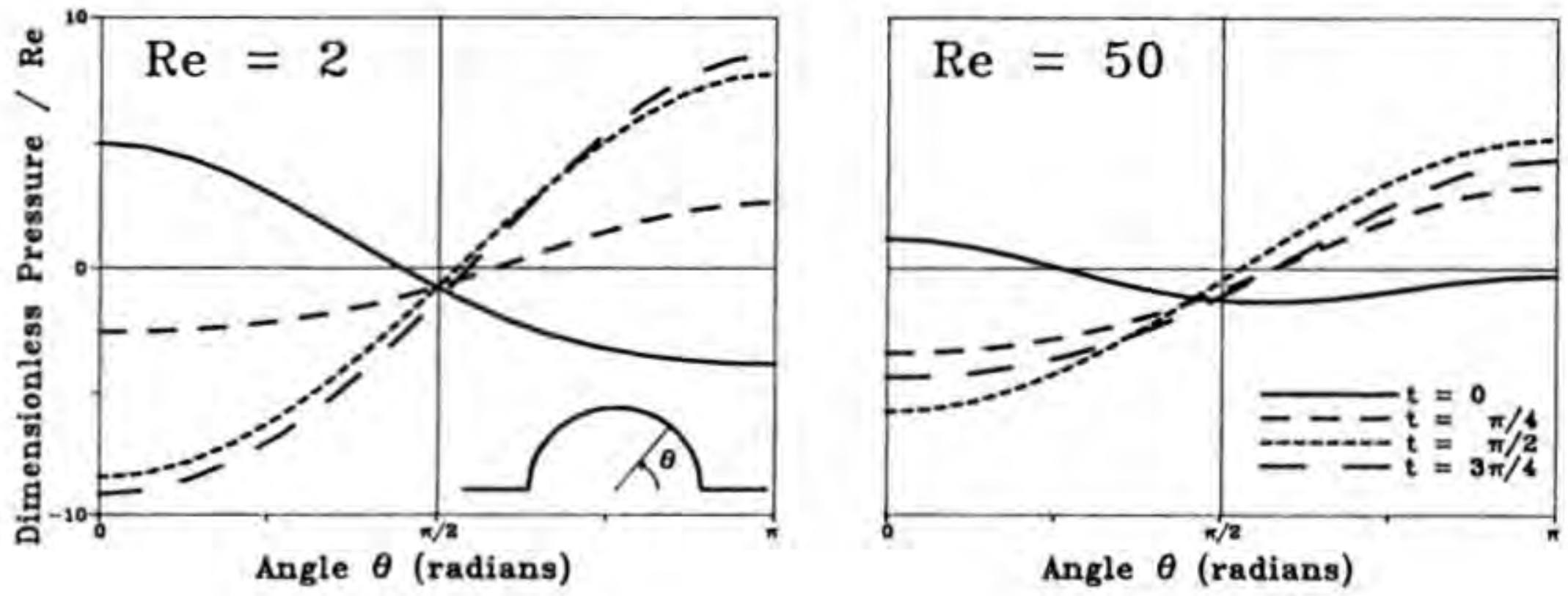

F 6 

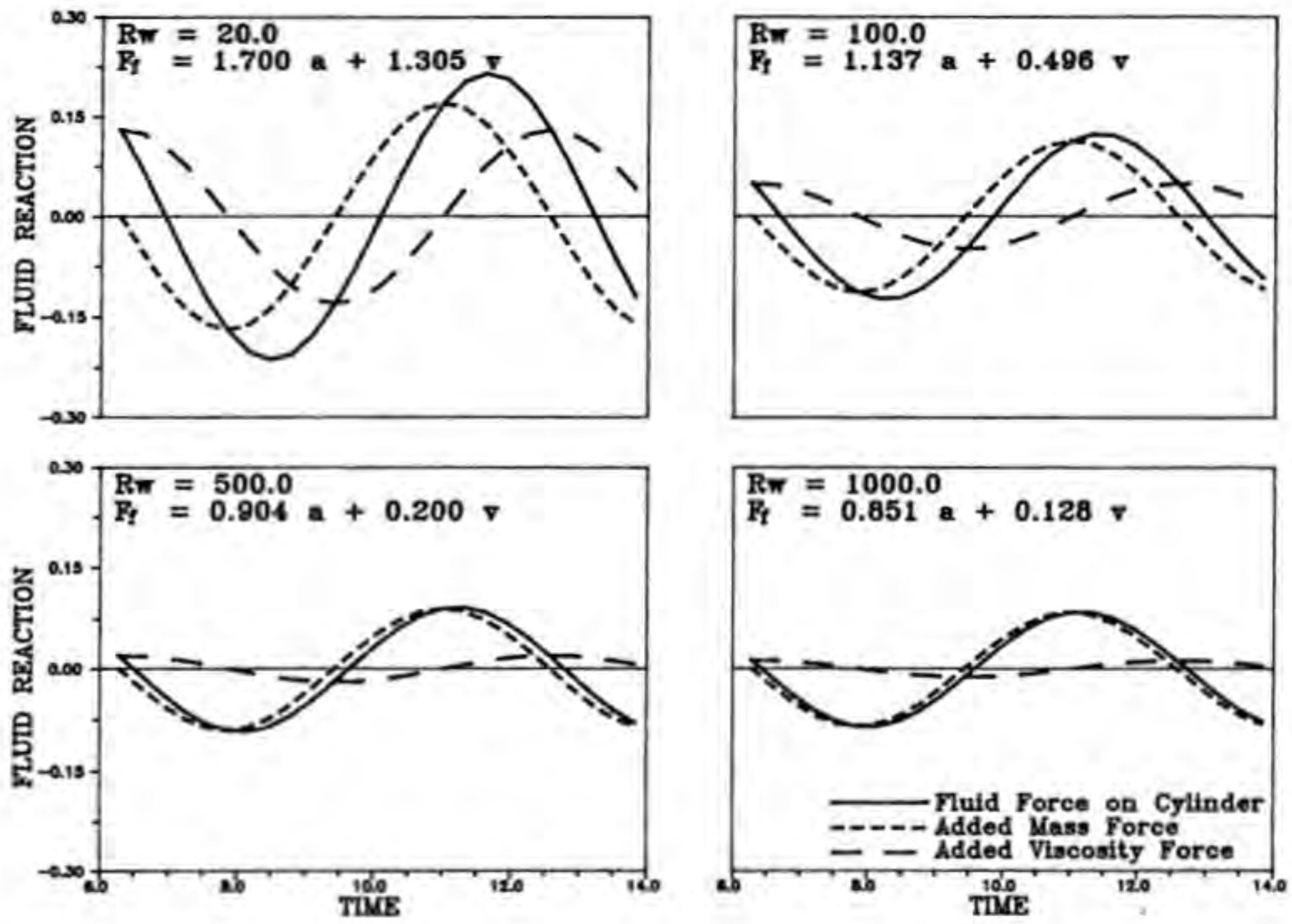


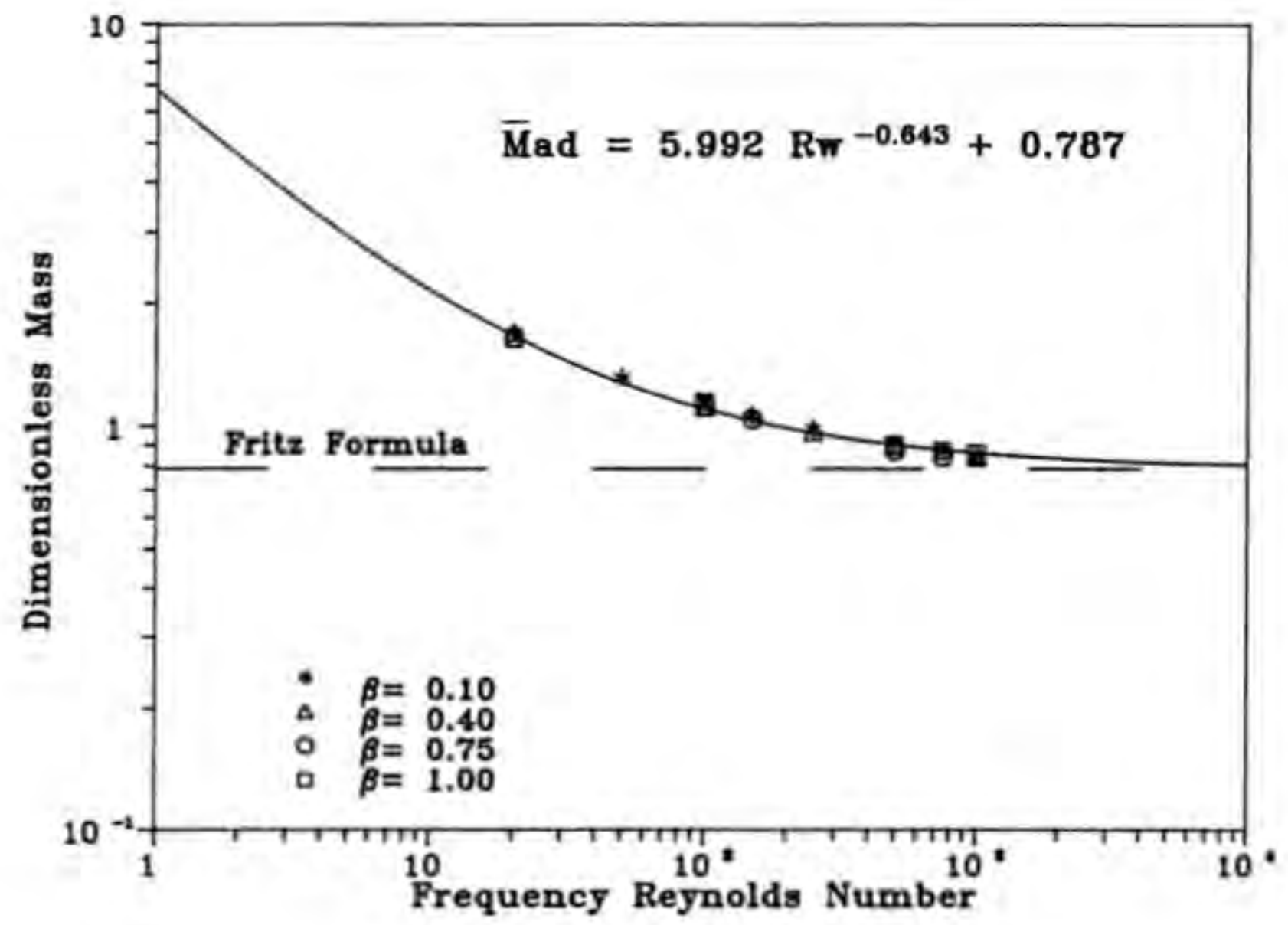




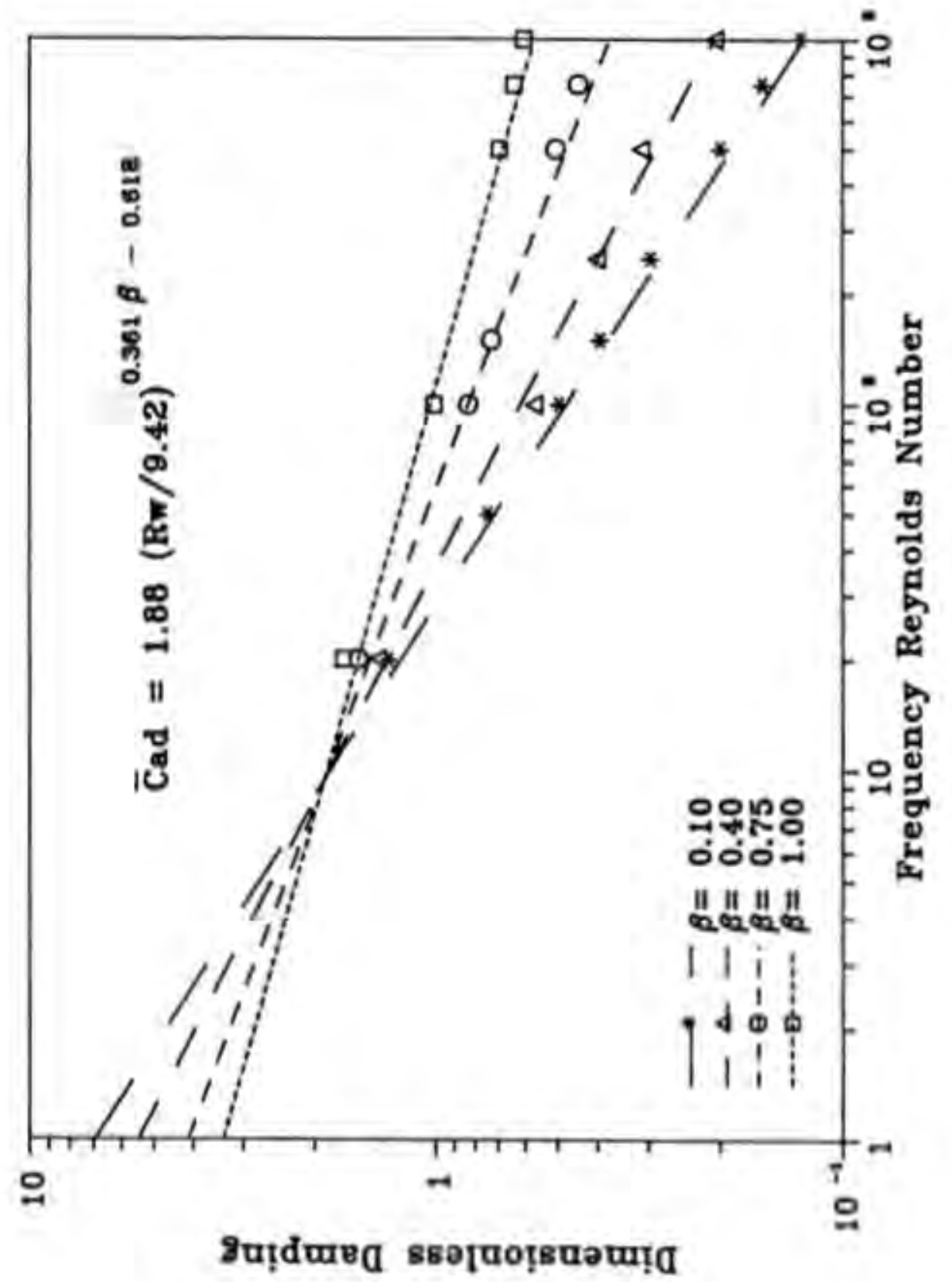

